\title{
Effect of Gln151 on L-phenylalanine feedback resistance of AroG isoform of DAHP synthase in Escherichia coli
}

\author{
Charintip Yenyuvadee, Nichaphat Kanoksinwuttipong, Kanoktip Packdibamrung* \\ Department of Biochemistry, Faculty of Science, Chulalongkorn University, Bangkok 10330 Thailand \\ *Corresponding author, e-mail: kanoktip.p@chula.ac.th
}

Received 6 Sep 2020

Accepted 17 Nov 2020

\begin{abstract}
In Escherichia coli, 3-deoxy-D-arabino-heptulosonate-7-phosphate synthase (DAHP synthase) is the first enzyme in aromatic amino acid synthetic pathway. It consists of three isoforms, AroG, AroF and AroH, which are inhibited by L-phenylalanine, L-tyrosine and L-tryptophan, respectively. AroG, the major isoform, represents about $80 \%$ of the total DAHP synthase activity in the cells. To increase the production of L-phenylalanine in $E$. coli, it is important to reveal the inhibition mechanism of AroG and relieve the degree of inhibition. Therefore, this study aims to investigate the important amino acid residue that interacts with L-phenylalanine at the regulatory site and determine its effect on L-phenylalanine feedback inhibition. From the previously reported 3D structure of AroG co-crystallized with L-phenylalanine, Gln151 was mutated to construct L-phenylalanine feedback resistant AroG. Three mutant clones (AroG ${ }^{\mathrm{Q} 151 \mathrm{~A}}$, AroG ${ }^{\mathrm{Q} 151 \mathrm{~L}}$ and $A \mathrm{AroG}^{\mathrm{Q} 151 \mathrm{~N}}$ ) were prepared by using QuikChange site-directed mutagenesis. The mutated enzymes exhibited a slightly higher DAHP synthase activity than the wild type, and a decrease in percentage of inhibition by $20 \mathrm{mM}$ L-phenylalanine from $51 \%$ to $12-27 \%$. The results suggested that H-bonding between $\mathrm{Gln} 151$ of AroG and the inhibitor, L-phenylalanine, had a high impact on L-phenylalanine feedback inhibition. aro $G^{\mathrm{Q} 151 \mathrm{~N}}$ is the good candidate to co-express with genes encoding key enzymes in aromatic amino acid synthetic pathway to obtain the L-phenylalanine overproducing E. coli strain.
\end{abstract}

KEYWORDS: L-phenylalanine, AroG, DAHP synthase, feedback inhibition

\section{INTRODUCTION}

L-Phenylalanine is one of the most important aromatic amino acids for humans and animals [1-3]. It is widely used in food and feed industries as, for example, supplementary food and precursor for low calorie sweetener aspartame [4-6]. L-Phenylalanine is also used in pharmaceutical industries for production of pharmaceutically active compounds like HIV protease inhibitor, antiinflammatory drugs [7], phenylethylamine [8], and catecholamines [9]. Moreover, L-phenylalanine, in combination with UV-C, is used for the production of mulberroside A like resveratrol and rutin [10]; or with UV-A therapy for the treatment of vitiligo [11]. Currently, L-phenylalanine is increasingly in great demand for the production of low-calorie sweetener, aspartame $[12,13]$. In early industrial processes, L-phenylalanine was produced by chemical synthesis, which led to various problems and was costly. The synthesis is gradually being replaced with bioprocessing, such as microbial fermentation and enzymatic transformation [14]. More recently, metabolic engineering in Escherichia coli has been focused because the main metabolisms of $E$. coli have enabled the introduction of such genetic modifications [15]. Furthermore, genetic engineering technique has been used as another approach to enhance L-phenylalanine yield [16].

The aromatic amino acid biosynthesis pathway in $E$. coli begins with the condensation of phosphoenolpyruvate (PEP) and erythrose 4-phosphate (E4P) to form 3-deoxy-D-arabino-heptulosonate-7phosphate through the catalysis of DAHP synthase (EC 4.1.2.15). DAHP synthase is the primary target for metabolic regulation by feedback inhibition since each of the three DAHP synthase isozymes is sensitive to regulation by the individual aromatic amino acid end products, L-phenylalanine, L-tyrosine or L-tryptophan [17-19]. About 80\% of total DAHP synthase activity is due to AroG, L-phenylalanine-sensitive isozyme. Therefore, the AroG, which is resistant to L-phenylalanine, should solve the mechanism of feedback inhibition and 
leads to overproduction of aromatic amino acids through fermentation.

In regard to the 3D structure of AroG cocrystallized with the substrate PEP determined by Shumilin and coworkers in 1999 [20], Hu and colleagues investigated the feedback inhibition site of AroG by amino acid replacement at Phe144, Pro150, Leu175, Leu179, Phe209, Trp215, and Val221. The enzyme activity assay showed that the mutated enzymes completely or partially relieved feedback inhibition of AroG addressed by L-phenylalanine [21].

In 2002, Shumilin and coworkers determined the crystal structure at $2.8 \AA$ of AroG in complex with its inhibitor, L-phenylalanine, PEP, and metal cofactor, $\mathrm{Mn}^{2+}$. Aromatic ring of L-phenylalanine is surrounded by hydrophobic side-chains of Pro150, Gln151, Leu175, Leu179, Phe209, Ser211, Val221, Ile10* (* represents amino acid residue of the companion subunit of the tight dimer), Ile13* and by main-chain atom of Met147, Ile148 and Val212. The carboxylate group of L-phenylalanine is coordinated by $\mathrm{O}$ and $\mathrm{N}$ of Ser180 and $\mathrm{N}$ of Asp7*, while the amino group of the inhibitor interacts with the sidechain oxygen atom of Gln151, as well as Asp6* and Asp7* [22]. In this study, Gln151 was selected for site-directed mutagenesis to determine the effect on L-phenylalanine feedback inhibition of AroG in the attempt to increase L-phenylalanine production.

\section{MATERIALS AND METHODS}

Selection of the amino acid residue at L-phenylalanine binding site for mutagenesis

The crystal structure of AroG from E. coli complexed with $\mathrm{Mn}^{2+}$, PEP, and L-phenylalanine in the Protein Data Bank with the code 1KFL was used for analysing the amino acid residues that interact with L-phenylalanine at the regulatory site of AroG. BIOVIA Discovery Studio 2020 software (Accelrys Co., Ltd., USA) was used to display the structure.

\section{Plasmids, bacterial strains and culture conditions}

All E. coli strains and plasmids used in this study are described in Table S1. E. coli Top10 was applied for molecular cloning. E. coli strains were cultured at $37^{\circ} \mathrm{C}$ in $\mathrm{LB}$ medium. Antibiotic (kanamycin $30 \mu \mathrm{g} / \mathrm{ml}$ ) was added to maintain the plasmids.

\section{Cloning of aroG}

The aroG (1053 bp) was amplified from genomic DNA of E. coli JM109 using forward primer (F_AroG_NcoI) containing NcoI site and reverse

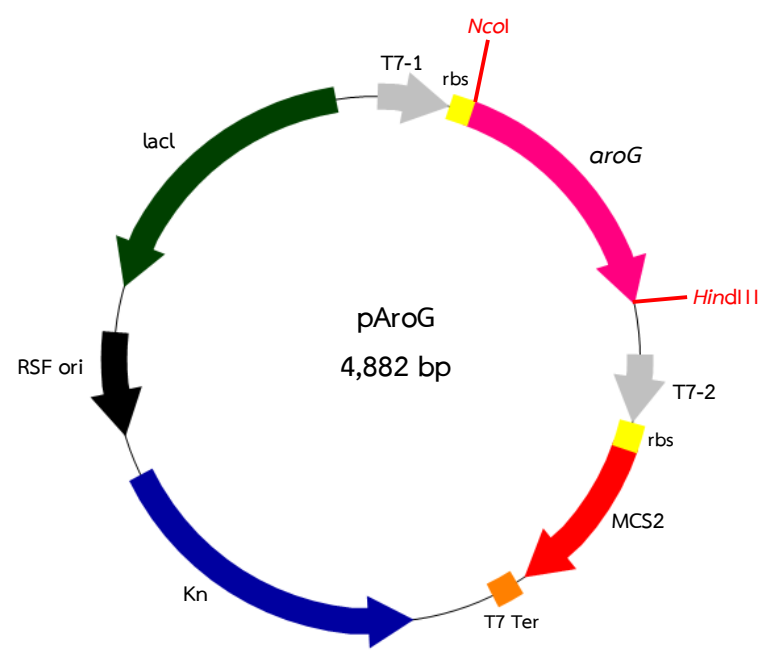

Fig. 1 Map of pAroG. aroG from E. coli JM109 was inserted into pRSFDuet-1 at NcoI and HindIII sites.

primer (R_AroG_HindIII) containing HindIII site. The PCR product was cloned into pRSFDuet-1 vector and then transformed into $E$. coli Top 10 by electroporation. The recombinant plasmid was confirmed by digestion with $\mathrm{NcoI}$ and HindIII. The nucleotide sequencing was performed by Bioneer Inc. (Korea) using ACYCDuetUP1 and DuetDOWN1 primers (Table S2).

Site directed mutagenesis and construction of pAroG $^{\text {wt }}$ and pAroG ${ }^{\text {fbr }}$

The L-phenylalanine feedback resistant aroG $\left(\right.$ aro $^{\mathrm{fbr}}{ }^{\mathrm{fr}}$ mutants, Q151A, Q151L and Q151N, were constructed by QuikChange site directed mutagenesis. Each aroG mutant gene was amplified using pAroG template and the primers shown in Table S2. After that, the DpnI digestion was performed to cut parental methylated DNA. The plasmids harboring the mutation site ( $\mathrm{pAroG}^{\mathrm{fbr}}$ : pAroG $^{\mathrm{Q} 151 \mathrm{~A}}$, pAroG ${ }^{\mathrm{Q} 151 \mathrm{~L}}$, and $\mathrm{pAroG}^{\mathrm{Q} 151 \mathrm{~N}}$ ) were verified by the nucleotide sequencing (Bioneer Inc., Korea). The pAroG ${ }^{\mathrm{wt}}$ and $\mathrm{pAroG}^{\mathrm{fbr}}$ were transformed into E. coli BL21(DE3) and selected on LB agar containing kanamycin $30 \mu \mathrm{g} / \mathrm{ml}$. The plasmid of each clone was extracted and confirmed by NcoI and HindIII digestion. Map of pAroG is shown in Fig. 1.

\section{Expression of aroG}

The single colonies of pAroG ${ }^{\mathrm{wt}}$ and $\mathrm{pAroG}^{\mathrm{fbr}}$ were separately cultured in $5 \mathrm{ml} \mathrm{LB}$ medium containing 
$30 \mu \mathrm{g} / \mathrm{ml}$ of kanamycin and then incubated with shaking at $37^{\circ} \mathrm{C}$ for $16-18 \mathrm{~h}$. A starter was made by inoculating $5 \%(\mathrm{v} / \mathrm{v})$ of each culture into $50 \mathrm{ml}$ of the same medium and conditions. For shake flask cultivation, the $5 \%(\mathrm{v} / \mathrm{v})$ of starters were transferred into $200 \mathrm{ml}$ of LB medium and incubated with shaking at $37^{\circ} \mathrm{C}$. After the $\mathrm{OD}_{600}$ reached 0.6 (log phase), expression of aro $G$ was induced by $1 \mathrm{mM}$ isopropyl$\beta$-D-thiogalactoside (IPTG) for $2 \mathrm{~h}$. The cells of each mutant clone were collected by centrifugation at $8000 \times \mathrm{g}$ for $10 \mathrm{~min}$ and resuspended in $5 \mathrm{ml}$ of resuspend buffer $(0.1 \mathrm{mM}$ potassium phosphate buffer (KPB), pH 7.0, $0.2 \mathrm{mM}$ PEP, $0.5 \mathrm{mM} \mathrm{1,4-}$ dithiothreitol, $0.1 \mathrm{mM}$ phenylmethylsulfonyl fluoride, and $10 \mathrm{mM}$ ethylenediaminetetraacetic acid). The cell pellet of each clone was dissolved in resuspend buffer, and cells were broken by ultrasonic cell disruption. The crude extracts were centrifuged at $10000 \times g$ for 20 min to collect the supernatants for dialysis. After dialysis, $1.5 \mathrm{ml}$ of crude extracts was centrifuged at $10000 \times g$ for $20 \mathrm{~min}$ to collect the supernatant for assay of enzyme activity and SDSPAGE analysis.

\section{DAHP synthase activity assay}

DAHP synthase activity was determined by the method modified from Schoner and Liu [23, 24]. The reaction mixture contained $50 \mathrm{mM} \mathrm{KPB}, \mathrm{pH} 6.5$, $5 \mathrm{mM}$ PEP, $2 \mathrm{mM}$ E4P, 0-20 mM L-phenylalanine, crude enzyme, and $\mathrm{H}_{2} \mathrm{O}$ in a total volume of $33.75 \mu \mathrm{l}$. The mixture was incubated at $30^{\circ} \mathrm{C}$ for $10 \mathrm{~min}$. The reaction was initiated when the enzyme was added and stopped by addition of $180 \mu \mathrm{l}$ of $10 \%$ $(\mathrm{w} / \mathrm{v})$ trichloroacetic acid. After that, the $45 \mu \mathrm{l}$ of $25 \mathrm{mM}$ sodium periodate in $62.5 \mathrm{mM}$ sulfuric acid was added to the mixture and incubated at $37^{\circ} \mathrm{C}$ for $30 \mathrm{~min}$. Then, $45 \mu \mathrm{l}$ of $2 \%(\mathrm{w} / \mathrm{v})$ sodium sulfate in $0.5 \mathrm{M}$ hydrochloric acid was rapidly mixed to stop the reaction; and $450 \mu \mathrm{l}$ of $0.36 \%(\mathrm{w} / \mathrm{v})$ thiobarbituric acid was added and mixed. The reaction mixture was boiled for $20 \mathrm{~min}$ and then cooled at room temperature. The absorbance at $549 \mathrm{~nm}$ was measured by a spectrophotometer. Protein concentration was determined by Lowry's method [25].

\section{SDS-PAGE analysis}

The expression of aro $G$ was confirmed by SDS-PAGE analysis. The slab gel solution consisted of $12.5 \%$ separating gel and 5\% stacking gel. For protein loading preparations, the crude enzymes of AroG ${ }^{\mathrm{wt}}$ and $A r o G^{\mathrm{fbr}}$ were mixed with $5 \mathrm{x}$ sample buffer (312.5 mM Tris- $\mathrm{HCl} \mathrm{pH}$ 6.8, 50\% (v/v) glycerol and $1 \%(\mathrm{w} / \mathrm{v})$ bromophenol blue) and boiled for
15 min. After that, the pellets were eliminated by centrifugation at $10000 \times g$ for $15 \mathrm{~min}$. The supernatants containing about $100 \mu \mathrm{g}$ protein were used for SDS-PAGE.

\section{RESULTS AND DISCUSSION}

\section{The amino acid residues at L-phenylalanine binding site}

The amino acid residues that interact with $\mathrm{L}-$ phenylalanine at the regulatory site of AroG are displayed in Fig. 2a. Hydrophobic side chain of L-phenylalanine interacts with Gln151, Leu175, Leu179, Phe209 Ser211, and Ile13* by van der Waals interactions, as well as Pro150, Val221 and Ile10* by pi-alkyl interactions. The carboxylate group of L-phenylalanine forms two H-bonds with Ser180, one H-bond with Asp7* and salt bridge interaction with Arg40; while the amino group forms one $\mathrm{H}$-bond with $\mathrm{G} \ln 151$ as well as salt bridge interactions with Asp6* and Asp7*. Among these amino acid residues in the regulatory site, Ser180, Gln151, Asp6*, and Asp7*, which can form H-bonds with L-phenylalanine, are interesting.

Ger et al [26] performed random mutagenesis of the cloned aroG on a plasmid vector and found that substitution of Ser180 to Phe relieved the degree of feedback inhibition from approximately $60 \%$ to less than $10 \%$ in the presence of $20 \mathrm{mM}$ L-phenylalanine. Replacement of Phe180 with Ser, Asn and Cys by site-directed mutagenesis demonstrated that Ser180 is a critical residue in the feedback inhibition of AroG.

Recently, Chen et al [27] used a method which integrates CRISPR/Cas9-facilitated engineering of the target gene(s) with growth-coupled and sensorguided in vivo screening (CGSS) to obtain variants of AroG $^{\text {fbr }}$ with increased resistance to feedback inhibition of L-phenylalanine. Two residues, Asp6 and Asp7, involved in the L-phenylalanine binding site of AroG were chosen for saturated mutagenesis. They successfully identified several novel Lphenylalanine-resistant AroG variants which exhibited higher specific DAHP synthase activity than that of the reference variant S180F at the presence of $40 \mathrm{mM}$ L-phenylalanine. The replacement of AroG $^{\mathrm{S} 180 \mathrm{~F}}$ with AroG ${ }^{\mathrm{D} 6 \mathrm{G}-\mathrm{D} 7 \mathrm{~A}}$, which gave the highest specific activity in the L-tryptophan-producing strain, significantly improved the L-tryptophan production by $38.5 \%(24.03 \pm 1.02 \mathrm{~g} / \mathrm{l}$ at $36 \mathrm{~h})$ in simple fed-batch fermentation.

In 2003, Hu et al [21] investigated the impact of Leu175 on L-phenylalanine feedback inhibition 

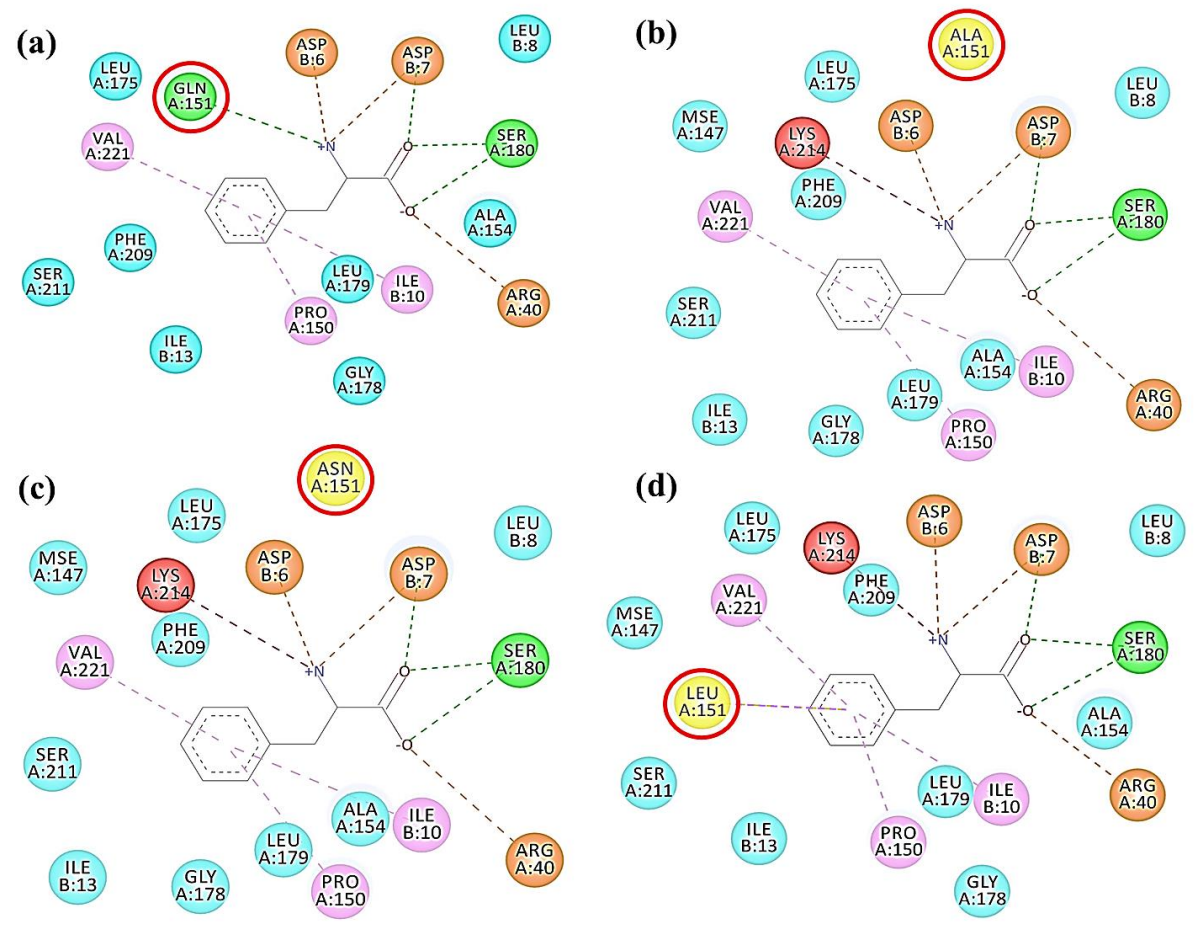

Fig. 2 Amino acid residues that interact with L-phenylalanine at the regulatory site of AroG (a) AroG ${ }^{\mathrm{wt}}$, (b) AroG ${ }^{\mathrm{Q} 151 \mathrm{~A}}$, (c) $A_{r o G}{ }^{\mathrm{Q} 151 \mathrm{~N}}$, (d) AroG ${ }^{\mathrm{Q} 151 \mathrm{~L}}$. Red circle represents Q151 and equivalent residues in the mutated enzymes. Yellow color refers to the mutated amino acid residues. Blue, pink, green, red, and orange colors represent the atoms which interact with L-phenylalanine using van der Waals interaction, pi-alkyl interaction, conventional hydrogen bonding, unfavorable donor-donor interaction as well as salt bridge and attractive charge, respectively. The display was made by using the BIOVIA Discovery Studio 2020 software.

by replacement with Ala, Gln and Asp. They concluded that L175D was mostly resistant to feedback inhibition. L175D enzyme elevated specific enzyme activity at $0 \mathrm{mM} \mathrm{L}$-phenylalanine from $2.70 \mathrm{U} / \mathrm{mg}$ of wild type to $4.46 \mathrm{U} / \mathrm{mg}$ and increased of relative enzymatic activity at $1 \mathrm{mM}$ L-phenylalanine from $8.2 \%$ to $83.5 \%$. Surprisingly, in our simulation only van der Waals interaction was found between Leu175 and L-phenylalanine.

In the present study, we are interested in Gln151 because it can form H-bond with L-phenylalanine as well as Ser180, Asp6 and Asp7. No mutation of this residue has never before been reported. Moreover, the pocket accommodating the aromatic ring of Lphenylalanine is formed by hydrophobic side-chains including that of $G \ln 151$. To investigate this amino acid residue, structures of AroG when Gln151 was substituted by Ala, Asn and Leu were simulated. We found that all replaced amino acids cannot form H-bond with L-phenylalanine (Fig. 2(b,c,d)). In addition, unfavorable donor-donor interaction occurred between amino groups of L-phenylalanine and Lys214 of all mutated enzymes. Differ from Ala and Asn, hydrophobic interaction between Leu151 and L-phenylalanine was detected.

\section{Construction of $\mathrm{pAroG}^{\mathrm{wt}}$ and $\mathrm{pAroG} \mathrm{G}^{\mathrm{fbr}}$}

The aro $G$ was cloned into pRSFDuet- 1 vector and then three aroG mutants: Q151A, Q151L and Q151N, were constructed. The recombinant plasmids were confirmed by NcoI and HindIII digestion. Two bands of DNA fragments were observed at 3800 bp and 1100 bp (Fig. 3). DNA sequencing indicated that the sequences of all inserted fragments were correct.

\section{Overexpression of AroG proteins}

$\mathrm{pAroG}^{\mathrm{wt}}$ and $\mathrm{pAroG} \mathrm{G}^{\mathrm{fbr}}$ were transformed into $E$. coli BL21(DE3). The transformants were cultured in LB medium and induced with $1 \mathrm{mM}$ IPTG for $2 \mathrm{~h}$. After overexpression, the crude extracts were dialysed to eliminate small molecular weight substances. Then, the expression of $\operatorname{aro} G^{\mathrm{wt}}$ and $\operatorname{aro} G^{\mathrm{fbr}}$ under T7 promoter was evaluated by SDS-PAGE 


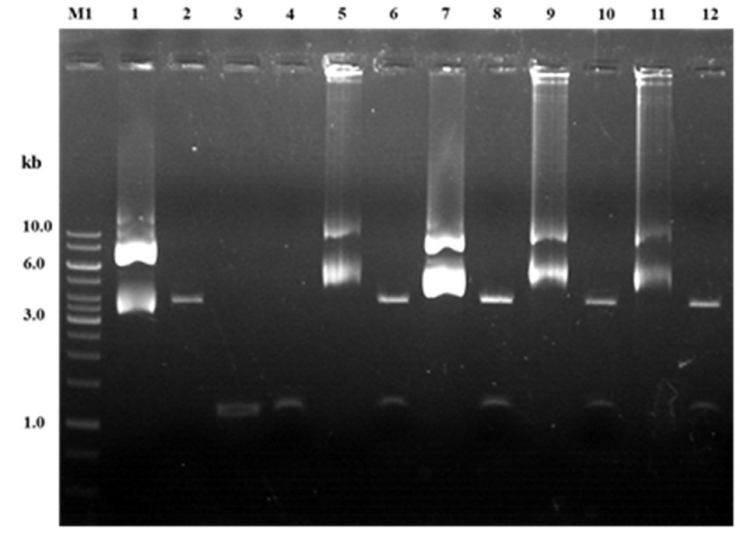

Fig. $3 \mathrm{NcoI}$ and HindIII digestion patterns of $\mathrm{pAroG}^{\mathrm{wt}}$ and pAroG $^{\mathrm{fbr}}$. Lane M1: Gene Ruler $1 \mathrm{~kb}$ DNA ladder, Lane 1: uncut pRSFDuet-1, Lane 2: NcoI and HindIII digested pRSFDuet-1, Lane 3: PCR product of aroG, Lane 4: PCR product of aroG digested with $N c o$ I and HindIII, Lane 5: uncut pAroG ${ }^{\mathrm{wt}}$, Lane 6: pAroG $^{\mathrm{wt}}$ digested with NcoI and HindIII. Lane 7: uncut pAroG ${ }^{\mathrm{Q} 151 \mathrm{~A}}$, Lane 8: pAroG ${ }^{\mathrm{Q} 151 \mathrm{~A}}$ digested with $N$ coI and HindIII. Lane 9: uncut pAroG ${ }^{\mathrm{Q} 151 \mathrm{~L}}$, Lane 10: pAroG $^{\mathrm{Q} 151 \mathrm{~L}}$ digested with NcoI and HindIII. Lane 11: uncut pAroG ${ }^{\mathrm{Q} 151 \mathrm{~N}}$, Lane 12: pAroG $^{\mathrm{Q} 151 \mathrm{~N}}$ digested with NcoI and HindIII.

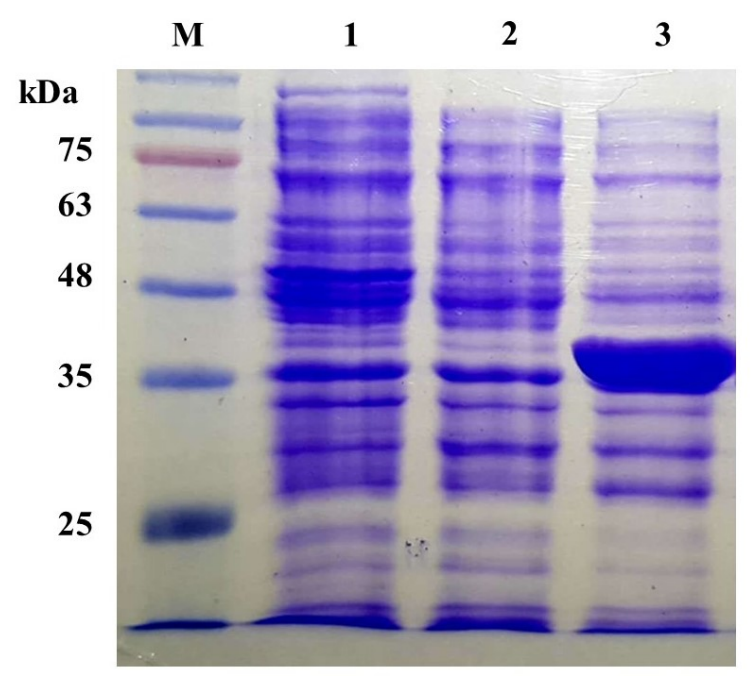

Fig. 4 SDS-PAGE analysis of recombinant AroG in E. coli. Lane M, TriColor Protein Ladder (10-180 kDa); Lane 1-3: crude extracts of E. coli BL21(DE3), E. coli BL21(DE3) harboring pRSFDuet-1, and E. coli BL21(DE3) harboring pAroG, respectively.

analysis. As shown in Fig. 4, the crude extracts of E. coli BL21(DE3) and E. coli BL21(DE3) harboring

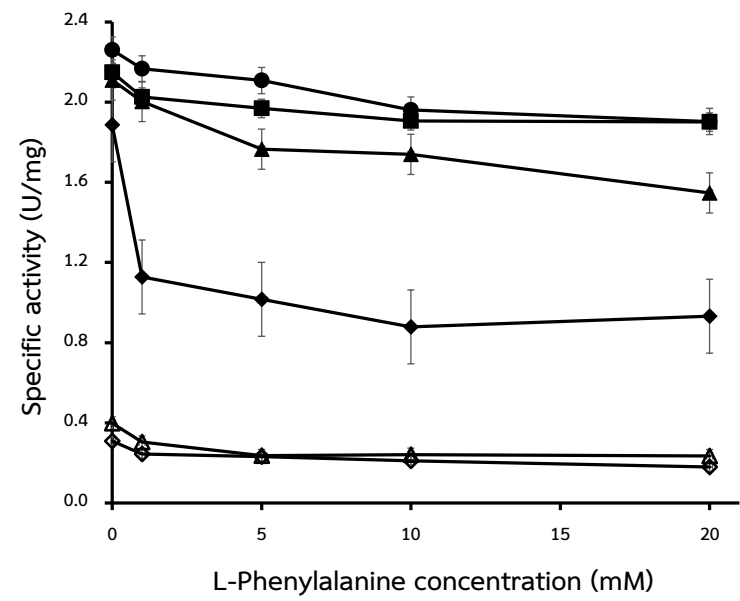

Fig. 5 L-Phenylalanine inhibition patterns of DAHP synthase activities. $(\diamond:$ E. coli BL21(DE3), $\triangle$ : E. coli BL21(DE3) harboring pRSFDuet-1, $\bullet$ : E. coli BL21(DE3) harboring pAroG $^{\mathrm{wt}}, \mathbf{\Delta}$ : E. coli BL21(DE3) harboring pAroG $^{\mathrm{Q} 151 \mathrm{~A}}$, $\mathbf{\square}$ : E. coli BL21(DE3) harboring pAroG ${ }^{\mathrm{Q} 151 \mathrm{~L}}$ and ๑: E. coli BL21(DE3) harboring $\left.\mathrm{pAroG}^{\mathrm{Q} 151 \mathrm{~N}}\right)$. The data were obtained from three independent experiments.

pRSFDuet-1 were used as a control (lanes 1 and 2). The band of the recombinant protein was detected after $1 \mathrm{mM}$ IPTG induction for $2 \mathrm{~h}$ (lane 3 ). The size of recombinant protein was approximately $38 \mathrm{kDa}$.

\section{DAHP synthase activity}

DAHP synthase activity of crude extracts from AroG mutants were assayed for sensitivity to feedback inhibition by L-phenylalanine at concentrations from $0 \mathrm{mM}$ to $20 \mathrm{mM}$. The results are shown in Fig. 5 . In the absence of L-phenylalanine, all Aro $\mathrm{G}^{\mathrm{fbr}}$ clones exhibited 12-20\% higher specific activities than that of $\mathrm{AroG}^{\mathrm{wt}}$. This phenomenon was also reported in L8F, S180F [26], F144A, L175A, L175Q, L175D, and F209A [21]. The activities of all recombinant enzymes were decreased in the same pattern when L-phenylalanine was added. All AroG mutants showed greater resistance to feedback inhibition when compared with AroG ${ }^{\mathrm{wt}}$. AroG ${ }^{\mathrm{Q} 151 \mathrm{~N}}$ gave the greatest inhibition pattern; while $\mathrm{AroG}^{\mathrm{Q} 151 \mathrm{~A}}$ mutant had the lowest inhibition pattern at 0 $20 \mathrm{mM}$ L-phenylalanine. Percentages of inhibition by $20 \mathrm{mM}$ L-phenylalanine were decreased from $51 \%$ of wild type to 12,16 and $27 \%$ for Q151L, Q151N and Q151A, respectively (Table 1). Destruction of two H-bonding between Ser180 and Lphenylalanine by substitution with Phe (S180F) was reported to decrease the percentage of inhibition 
Table 1 DAHP synthase activities and feedback inhibitions of various AroG clones.

\begin{tabular}{lccc}
\hline Mutant & $\begin{array}{c}\text { SA at } 0 \mathrm{mM} \\
\text { L-phenylalanine } \\
\text { (U/mg protein) }\end{array}$ & $\begin{array}{c}\text { SA at 20 mM } \\
\text { L-phenylalanine } \\
\text { (U/mg protein) }\end{array}$ & $\begin{array}{c}\text { Percentage of } \\
\text { inhibition at 20 mM } \\
\text { L-phenylalanine (\%) }\end{array}$ \\
\hline E. coli BL21(DE3) & $0.31 \pm 0.04$ & $0.18 \pm 0.05$ & 42 \\
pRSFDuet-1 & $0.40 \pm 0.07$ & $0.24 \pm 0.04$ & 41 \\
AroG & $1.89 \pm 0.39$ & $0.93 \pm 0.45$ & 51 \\
AroG $^{\mathrm{Q} 151 \mathrm{~A}}$ & $2.11 \pm 0.75$ & $1.55 \pm 0.41$ & 27 \\
AroG & $1.90 \pm 0.05$ & 12 \\
AroG & $2.15 \pm 0.11$ & $1.90 \pm 0.06$ & 16 \\
\hline
\end{tabular}

$\mathrm{SA}=$ specific activity.

by L-phenylalanine at concentration of $20 \mathrm{mM}$ from $58 \%$ to $7.4 \%$ [26].

In conclusion, the results suggested that $\mathrm{H}$ bonding between Gln151 of AroG and the inhibitor, L-phenylalanine, had a high impact on Lphenylalanine feedback inhibition. Thus, we can use Asn substitution at Gln151 of AroG to increase the enzymatic activity of DAHP synthase especially under high L-phenylalanine concentration.

\section{Appendix A. Supplementary data}

Supplementary data associated with this article can be found at http://dx.doi.org/10.2306/ scienceasia1513-1874.2021.004.

Acknowledgements: This work was partially supported by Sci Super II Grant to develop research potential of the Department of Biochemistry, Faculty of Science, Chulalongkorn University.

\section{REFERENCES}

1. Liu S, Zhang L, Mao J, Ding Z, Shi G (2015) Metabolic engineering of Escherichia coli for the production of phenylpyruvate derivatives. Metab Eng 32, 55-65.

2. Yakandawala N, Romeo T, Friesen A, Madhyastha S (2008) Metabolic engineering of Escherichia coli to enhance L-phenylalanine production. Appl Microbiol Biotechnol 78, 283-291.

3. Liu Y, Xu Y, Ding D, Wen J, Zhu B, Zhang D (2018) Genetic engineering of Escherichia coli to improve Lphenylalanine production. BMC Biotechnol 18, 1-12.

4. Wu Y, Jiang P, Fan C, Wang J, Shang L, Huang W (2003) Co-expression of five genes in E. coli for L-phenylalanine in Brevibacterium flavum. World $J$ Gastroenterol 9, 342-346.

5. Baez-vivoros JL, Osuna J, Hernandez-Chavez G, Soberon X, Bolivar F, Gosset G (2004) Metabolic engineering and protein directed evolution increase the yield of L-phenylalanine synthesized from glucose in Escherichia coli. Biotechnol Bioeng 87, 516-524.
6. Liu S, Liu R, Xiao M, Zhang L, Ding Z, Gu Z, Shi Y (2014) A systems level engineered E. coli capable of efficiently producing L-phenylalanine. Process Biochem 49, 751-757.

7. Weiner M, Albermann C, Gottlieb K, Sprenger GA, Weuster-Botz D (2014) Fed-batch production of Lphenylalanine from glycerol and ammonia with recombinant Escherichia coli. Biochem Eng J 83, 62-69.

8. Silkaitis RD, Mosnaim AD (1976) Pathways linking L-phenylalanine and 2-phenylethylamine with $p$ tyramine in rabbit brain. Brain Res 114, 105-115.

9. Khamduang M, Packdibamrung K, Chutmanop J, Chisti Y, Srinophakun P (2009) Production of L-phenylalanine from glycerol by a recombinant Escherichia coli. J Ind Microbiol Biotechnol 36, 1267-1274.

10. Pongkitwitoon B, Simpan K, Chobsri T, Sritularak B, Putalun W (2020) Combined UV-C irradiation and precursor feeding enhances mulberroside A production in Morus alba L. cell suspension cultures. ScienceAsia 46, 679-685.

11. Siddiqui A, Stolk L, Bhaggoe R, Hu R, Schutgens $\mathrm{R}$, Westerhof W (1994) L-Phenylalanine and UVA irradiation in the treatment of vitiligo. Dermatology 188, 215-218.

12. Ahmad A, David J (2017) Effect of different levels of low-calorie sweetener aspartame on sensory attributes of diabetic Rasgulla. J Pharm Innov 6, 82-84.

13. Zhou H, Liao X, Wang T, Du G, Chen J (2010) Enhanced L-phenylalanine biosynthesis by coexpression of $p h e A^{\mathrm{fbr}}$ and aroF $F^{\mathrm{wt}}$. Bioresour Technol 101, 4151-4156.

14. Zhang C, Zhang J, Kang Z, Du G, Yu X, Wang T, Chen $\mathrm{J}$ (2013) Enhanced production of L-phenylalanine in Corynebacterium glutamicum due to the introduction of Escherichia coli wild-type gene aroH. J Ind Microbiol Biot 40, 643-651.

15. Ikeda M (2006) Towards bacterial strains overproducing L-tryptophan and other aromatics by metabolic engineering. Appl Microbiol Biotechnol 69, 615-626.

16. Wu W, Guo X, Zhang M, Huang Q, Qi F, Huang J (2018) Enhancement of L-phenylalanine production 
in Escherichia coli by heterologous expression of Vitreoscilla hemoglobin. Biotechnol Appl Biochem 65, 476-483.

17. Kikuchi Y, Tsujimoto K, Kurahashi O (1997) Mutational analysis of the feedback sites of phenylalanine-sensitive 3-deoxy-D-arabinoheptulosonate-7-phosphate synthase of Escherichia coli. Appl Environ Microbiol 63, 761-762.

18. McCandlis R, Poling M, Herrmann K (1978) 3-Deoxy$\mathrm{D}$-arabino-heptulosonate 7-phosphate synthase. $\mathrm{Pu}$ rification and molecular characterization of the phenylalanine-sensitive isoenzyme from Escherichia coli. J Biol Chem 253, 4259-4265.

19. Niu H, Li R, Liang Q, Qi Q, Li Q, Gu P (2019) Metabolic engineering for improving L-tryptophan production in Escherichia coli. J Ind Microbiol Biotechnol 46, 55-65.

20. Shumilin I, Kretsinger R, Bauerle R (1999) Crystal structure of phenylalanine-regulated 3-deoxyD-arabino-heptulosonate-7-phosphate synthase from Escherichia coli. Structure 7, 865-875.

21. Hu C, Jiang P, Xu Jian, Wu Y, Huang W (2003) Mutation analysis of the feedback inhibition site of phenylalanine-sensitive 3-deoxy-D-arabinoheptulosonate-7-phosphate synthase of Escherichia coli. J Basic Microbiol 43, 399-406.

22. Shumilin I, Zhao C, Bauerle R, Kretsinger R
(2002) Allosteric inhibition of 3-deoxy-D-arabinoheptulosonate-7-phosphate synthase alters the coordination of both substrates. J Mol Biol 320, 1147-1156.

23. Schoner R, Herrmann K (1976) 3-Deoxy-D-arabinoheptulosonate 7-phosphate synthase purification, properties, and kinetics of the tyrosine-sensitive isoenzyme from Escherichia coli. J Biol Chem 251, 5440-5447.

24. Liu Y, Li P, Zhao K, Wang B, Jiang C, Drake H, Liu S (2008) Corynebacterium glutamicum contains 3-deoxy-D-arabino-heptulosonate 7-phosphate synthases that display novel biochemical features. Appl Environ Microbiol 74, 5497-5503.

25. Lowry O, Rosebrough N, Farr A, Randall R (1951) Protein measurement with the folin phenol reagent. J Biol Chem 193, 265-275.

26. Ger Y, Chen S, Chiang H, Shiuan D (1994) A single Ser-180 mutation desensitizes feedback inhibition of the phenylalanine-sensitive 3-deoxy-D-arabinoheptulosonate 7-phosphate (DAHP) synthetase in Escherichia coli. J Biochem 116, 986-990.

27. Chen M, Chen L, Zeng A (2019) CRISPR/Cas9facilitated engineering with growth-coupled and sensor-guided in vivo screening of enzyme variants for a more efficient chorismate pathway in $E$. coli. Metab Eng Commun 9, ID e00094 


\section{Appendix A. Supplementary data}

Table S1 Bacterial strains and plasmids used in this study.

\begin{tabular}{|c|c|c|}
\hline Strain/Plasmid & Characteristic & Source/Reference \\
\hline \multicolumn{3}{|l|}{ Strain } \\
\hline E. coli BL21(DE3) & $\mathrm{F}-\mathrm{ompT} h s d S_{\mathrm{B}}\left(\mathrm{r}_{\mathrm{B}}-, \mathrm{m}_{\mathrm{B}}-\right)$ gal dcm (DE3) & Novagen \\
\hline E. coli JM109 & $\begin{array}{l}\mathrm{F}^{\prime} \text { traD36 } \text { pro }^{+} B^{+} \text {lacI } \\
\text { recA1 relA1 endA1 thi hsdR17 }\end{array}$ & Novagen \\
\hline E. coli Top 10 & $\begin{array}{l}\mathrm{F}-\text { mcrA } \Delta(\text { mrr-hsdRMS-mcrBC) } \varphi 80 \text { lacZ } \Delta \mathrm{M} 15 \Delta \text { lacX74 recA1 araD139 } \\
\Delta\left(\text { ara-leu) } 7697 \text { galU galK } \lambda-\operatorname{rpsL}\left(\mathrm{Str}^{\mathrm{R}}\right) \text { endA1 nup } \mathrm{G}\right.\end{array}$ & Invitrogen \\
\hline \multicolumn{3}{|l|}{ Plasmid } \\
\hline pRSFDuet-1 & pRSF1030 replicon, T7 promoter expression vector, lacI, Kan ${ }^{\mathrm{r}}$ & Novagen \\
\hline pAroG $^{\mathrm{wt}}$ & pRSFDuet-1 inserted with aro $G^{\mathrm{wt}}$ under the control of T7 promoter & This study \\
\hline pAroG ${ }^{\mathrm{Q} 151 \mathrm{~A}}$ & pRSFDuet- 1 inserted with $\operatorname{aro}^{\mathrm{Q} 151 \mathrm{~A}}$ under the control of T7 promoter & This study \\
\hline pAroG ${ }^{Q 151 L}$ & pRSFDuet-1 inserted with aro $G^{\mathrm{Q} 151 \mathrm{~L}}$ under the control of T7 promoter & This study \\
\hline pAroG ${ }^{\mathrm{Q} 151 \mathrm{~N}}$ & pRSFDuet-1 inserted with aro $G^{\mathrm{Q} 151 \mathrm{~N}}$ under the control of $\mathrm{T} 7$ promoter & This study \\
\hline
\end{tabular}

Table S2 The sequences of oligonucleotide primers for PCR amplification, DNA sequencing and site-directed mutagenesis in this study.

\begin{tabular}{|c|c|c|}
\hline Primer & Sequence & $T_{\mathrm{w}}\left({ }^{\circ} \mathrm{C}\right)$ \\
\hline \multicolumn{3}{|c|}{ For PCR amplification and DNA sequencing } \\
\hline F_AroG_NcoI & 5'-CATGCCATGGTGTATCAGAACGACGATTTACGCATCAAAGAAATC-3' & 61.4 \\
\hline R_AroG_HindIII & 5'-CCAAGCTTTTACCCGCGACGCGCTTTCACTGC-3’ & 67.2 \\
\hline ACYCDuetUP1 & 5'-GGATCTCGACGCTCTCCCT-3' & 60.0 \\
\hline DuetDOWN1 & 5'-GATTATGCGGCCGTGTACAA-3' & 57.0 \\
\hline \multicolumn{3}{|c|}{ For Site-directed mutagenesis } \\
\hline F_5'Q151A & 5'-GGTGAATTCCTCGATATGATCACTCCTGCCTATCTCGCTGACCTGATGAGCTGGGGC-3' & 75.4 \\
\hline R_3' Q151A & $5^{\prime}$-GCCCCAGCTCATCAGGTCAGCGAGATAGGCAGGAGTGATCATATCGAGGAATTCACC- $3^{\prime}$ & 75.4 \\
\hline F_5' Q151L & 5'-GGTGAATTCCTCGATATGATCACTCCTCTGTATCTCGCTGACCTGATGAGCTGGGGC-3' & 74.7 \\
\hline R_3' Q151L & 5'-GCCCCAGCTCATCAGGTCAGCGAGATACAGAGGAGTGATCATATCGAGGAATTCACC-3' & 74.7 \\
\hline F_5' Q151N & 5'-GGTGAATTCCTCGATATGATCACTCCTAATTATCTCGCTGACCTGATGAGCTGGGGC-3’ & 73.2 \\
\hline R_3' Q151N & 5'-GCCCCAGCTCATCAGGTCAGCGAGATAATTAGGAGTGATCATATCGAGGAATTCACC-3' & 73.2 \\
\hline
\end{tabular}

